\title{
Synthesis of a hexasaccharide fragment of the O-deacetylated GXM of C. neoformans serotype B
}

\author{
Wei Zhao and Fanzuo Kong* \\ Research Center for Eco-Environmental Sciences, Academia Sinica, PO Box 2871, Beijing 100085, China
}

Received 6 April 2004; accepted 15 April 2004

Available online 10 June 2004

\begin{abstract}
D-Xyl $p-(1 \rightarrow 4)-\alpha-\mathrm{D}-\mathrm{Man} p-(1 \rightarrow 3)-[\beta-\mathrm{D}-\mathrm{Xyl} p-(1 \rightarrow 2)]-\alpha-\mathrm{D}-\mathrm{Man} p-(1 \rightarrow 3)-[\beta-\mathrm{D}-\mathrm{Xyl} p-(1 \rightarrow 2)]-\alpha-\mathrm{D}-\mathrm{Man} p$, the fragment of the exopolysaccharide from Cryptococcus neoformans serovar B, was synthesized as its methyl glycoside. Thus, acetylation of allyl 3-O-benzoyl-4,6- $O$-benzylidene- $\alpha$-D-mannopyranoside (1) followed by debenzylidenation and selective 6-O-benzoylation afforded allyl 2- $O$-acetyl-3,6-di- $O$-benzoyl- $\alpha$-D-mannopyranoside (4). Glycosylation of 4 with 2,3,4-tri- $O$-benzoyl-D-xylopyranosyl trichloroacetimidate $(5)$ furnished the $\beta-(1 \rightarrow 4)$-linked disaccharide $\mathbf{6}$. Deallylation followed by trichloroacetimidate formation gave the disaccharide donor 8, and subsequent coupling with allyl 2,3,4-tri- $O$-benzoyl- $\beta$-D-xylopyranosyl-( $1 \rightarrow 2)-4,6$-di- $O$-benzoyl- $\alpha$-Dmannopyranoside (9), produced the tetrasaccharide 10. Reiteration of deallylation and trichloroacetimidate formation from $\mathbf{1 0}$ yielded the tetrasaccharide donor 12. The downstream disaccharide acceptor $\mathbf{1 8}$ was obtained by condensation of $\mathbf{5}$ with methyl 3-Oacetyl-4,6- $O$-benzylidene- $\alpha$-D-mannopyranoside, followed by debenzylidenation, benzoylation, and selective 3-O-deacetylation. Coupling of 18 with 12 afforded the hexasaccharide 19, and subsequent deprotection gave the hexasaccharide glycoside 20. Selective $2^{\prime \prime}$-O-deacetylation of $\mathbf{1 9}$ gave the hexasaccharide acceptor 21. Condensation of $\mathbf{2 1}$ with glucopyranosyluronate imidate $\mathbf{2 2}$ did not produce the expected heptasaccharide glycoside; instead, a transacetylation product $\mathbf{1 9}$ was obtained. Meanwhile, there was no reaction between $\mathbf{2 1}$ and the bromide donor $\mathbf{2 3}$.
\end{abstract}

(C) 2004 Elsevier Ltd. All rights reserved.

Keywords: Mannose; Xylose; Glucuronic acid

\section{Introduction}

Glucuronoxylomannan (GXM) as the major capsule component is produced from Cryptococcus neoformans, a primary cause of opportunistic infections associated with AIDS. ${ }^{1,2}$ Of the four major serotypes ${ }^{3}$ A-D for GXM, D has the simplest pentaose structure while $\mathrm{C}$ has the most complex octasaccharide structure. All the four serotypes are composed of a linear $\alpha-(1 \rightarrow 3)$-linked mannosyl backbone with $\beta$-glucopyranosyluronic acid, $\beta$-xylopyranosyl, and 6-O-acetyl substituents ${ }^{4}$ (Fig. 1).

The synthesis of trisaccharide and tetrasaccharide fragments ${ }^{5}$ corresponding to structures in capsular polysaccharides of $C$. neoformans and the synthesis of a

\footnotetext{
* Corresponding author. Tel.: +86-10-62936613; fax: +86-10-62923563; e-mail: fzkong@mail.rcees.ac.cn
}

pentasaccharide ${ }^{6}$ - the repeating unit of the polysaccharide in C. neoformans serovar D-have appeared. In our previous work, ${ }^{7}$ the successful syntheses of the hexasaccharide repeating unit of O-deacetylated GXM of $C$. neoformans serotype $\mathrm{A}$ and its frame-shifted hexasaccharide glycoside were reported. We now report a convergent synthesis of the hexasaccharide fragment of O-deacetylated GXM of C. neoformans serotype B, and a trial for the synthesis of the repeating unit of the serotype B.

\section{Results and discussion}

As outlined in Scheme 1, acetylation of allyl 3-O-benzoyl-4,6- $O$-isopropylidene- $\alpha$-D-mannopyranoside, ${ }^{8}$ (1) followed by debenzylidenation $(80 \%)$ and selective 6-Obenzoylation $(90 \%)$, gave the glycosyl acceptor 4 . 


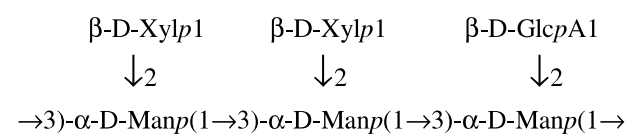

A

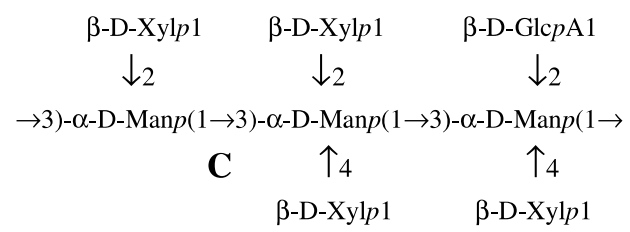

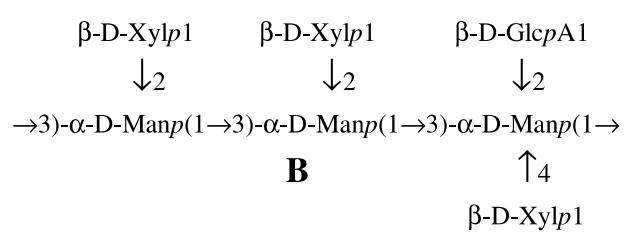

$\begin{array}{cc}\beta-\mathrm{D}-\mathrm{Xyl} p 1 & \beta-\mathrm{D}-\mathrm{Glc} p \mathrm{~A} 1 \\ \downarrow_{2} & \downarrow_{2} \\ \rightarrow 3)-\alpha-\mathrm{D}-\operatorname{Man} p(1 \rightarrow 3)-\alpha-\mathrm{D}-\operatorname{Man} p(1 \rightarrow 3)-\alpha-\mathrm{D}-\operatorname{Man} p(1 \rightarrow\end{array}$

D

Figure 1. Model structures of deacetylated GXM of C. neoformans serotypes A-D.

Condensation of 4 with 2,3,4-tri- $O$-benzoyl-D-xylopyranosyl trichloroacetimidate ${ }^{9}$ (5) afforded $\beta-(1 \rightarrow 4)$ linked disaccharide $\mathbf{6}(90 \%)$, and subsequent deallylation (85\%) with $\mathrm{PdCl}_{2}$ in methanol, and trichloroacetimidate formation ${ }^{10}$ produced the upstream disaccharide donor $8(90 \%)$. Coupling of 8 with allyl 2,3,4-tri- $O$-benzoyl- $\beta$ D-xylopyranosyl- $(1 \rightarrow 2)$-4,6-di- $O$-benzoyl- $\alpha$-D-mannopyranoside $(\mathbf{9})^{7}$ afforded the tetrasaccharide $10(75 \%)$, subsequent deallylation $(85 \%)$ and trichloroacetimidate formation $(90 \%)$ produced the tetrasaccharide donor $\mathbf{1 2}$.

The downstream disaccharide acceptor $\mathbf{1 8}$ was similarly prepared. Thus, selective 3-O-acetylation of methyl 4,6- $O$-benzylidene- $\alpha$-D-mannopyranoside (13) gave methyl 3- $O$-acetyl-4,6- $O$-benzylidene- $\alpha$-D-mannopyranoside (14) in satisfactory yield (80\%). Coupling of $\mathbf{1 4}$ with $5(80 \%)$ followed by debenzylidenation $(80 \%)$, benzoylation $(85 \%)$, and selective removal of the 3-O-acetyl group $(85 \%)$ by methanolysis ${ }^{11}$ with a mixture of Me$\mathrm{COCl}(2.0 \mathrm{~mL})$ in $\mathrm{CH}_{2} \mathrm{Cl}_{2}(10 \mathrm{~mL})$ and $\mathrm{MeOH}(40 \mathrm{~mL})$ produced the disaccharide acceptor 18.

Condensation of 18 with 12 successfully yielded the hexasaccharide glycoside $19(60 \%)$, and its deprotection in ammonia-saturated-methanol gave the free hexasaccharide fragment of C. neoformans serotype B.

A trial for the synthesis the heptasaccharide repeating unit of the serotype B was carried out. Thus, selective 2"O-deacetylation of $\mathbf{1 9}$ with a mixture of $\mathrm{MeCOCl}$ $(3.5 \mathrm{~mL})$ in $\mathrm{CH}_{2} \mathrm{Cl}_{2}(10 \mathrm{~mL})$ and $\mathrm{MeOH}(40 \mathrm{~mL})$ for 3 days afforded the hexasaccharide acceptor 21 in a fair yield $(60 \%)$. This indicated that there was a serious steric hindrance at C-2" position as the concentration of $\mathrm{MeCOCl}$ used was relatively higher, the reaction time was longer, but the yield was lower compared to selective deacetylation of $\mathbf{1 7}$.

Reaction of 21 with methyl 2,3,4-tri- $O$-acetyl- $\alpha$-Dglucopyranosyluronate trichloroacetimidate (22) under the coupling conditions did not give the expected heptasaccharide glycoside. Instead, acetyl transferring occurred giving 19 as the product. Meanwhile, reaction between 21 and methyl 2,3,4-tri- $O$-acetyl- $\alpha$-D- glucopyranosyluronate bromide (23) did not occur at all. Thus, the synthesis of the repeating unit of $C$. neoformans serotype B should be tried in other different ways.

In summary, a convergent synthesis of the hexasaccharide fragment of $C$. neoformans serotype B was achieved, but the strategy presented here could not be used for the synthesis of the repeating unit of GXM of C. neoformans serotype B.

\section{Experimental}

\subsection{General methods}

Melting points were determined with a 'Mel-Temp' apparatus. Optical rotations were determined with a Perkin-Elmer model 241-MC automatic polarimeter for solutions in a $1-\mathrm{dm}$ jacketed cell. ${ }^{1} \mathrm{H}$ NMR and ${ }^{13} \mathrm{C}$ NMR spectra were recorded with Varian XL-400 and Varian XL-200 spectrometers, for solutions in $\mathrm{CDCl}_{3}$ or in $\mathrm{D}_{2} \mathrm{O}$ as indicated. Chemical shifts are expressed in ppm downfield from the $\mathrm{Me}_{4} \mathrm{Si}$ resonance. Mass spectra were recorded with a VG PLATFORM mass spectrometer using the ESI mode. Thin-layer chromatography (TLC) was performed on silica gel HF with detection by charring with $30 \%(\mathrm{v} / \mathrm{v})$ sulfuric acid in $\mathrm{MeOH}$ or by UV detection. Column chromatography was conducted by elution of a column $(8 \times 100 \mathrm{~mm}$, $16 \times 240 \mathrm{~mm}, 18 \times 300 \mathrm{~mm}, 35 \times 400 \mathrm{~mm})$ of silica gel (100-200 mesh) with EtOAc-petroleum ether (bp 60$90^{\circ} \mathrm{C}$ ) as the eluent. Analytical LC was performed with a Gilson HPLC consisting of a pump (model 306), stainless-steel column packed with silica gel (Spherisorb $\mathrm{SiO}_{2}, 10 \times 300 \mathrm{~mm}$ or $4.6 \times 250 \mathrm{~mm}$ ), differential refractometer (132-RI detector), UV-vis detector (model 118). EtOAc-petroleum ether (bp $60-90^{\circ} \mathrm{C}$ ) was used as the eluent at a flow rate of $1-4 \mathrm{~mL} / \mathrm{min}$. Solutions were concentrated at a temperature $<60^{\circ} \mathrm{C}$ under diminished pressure. 

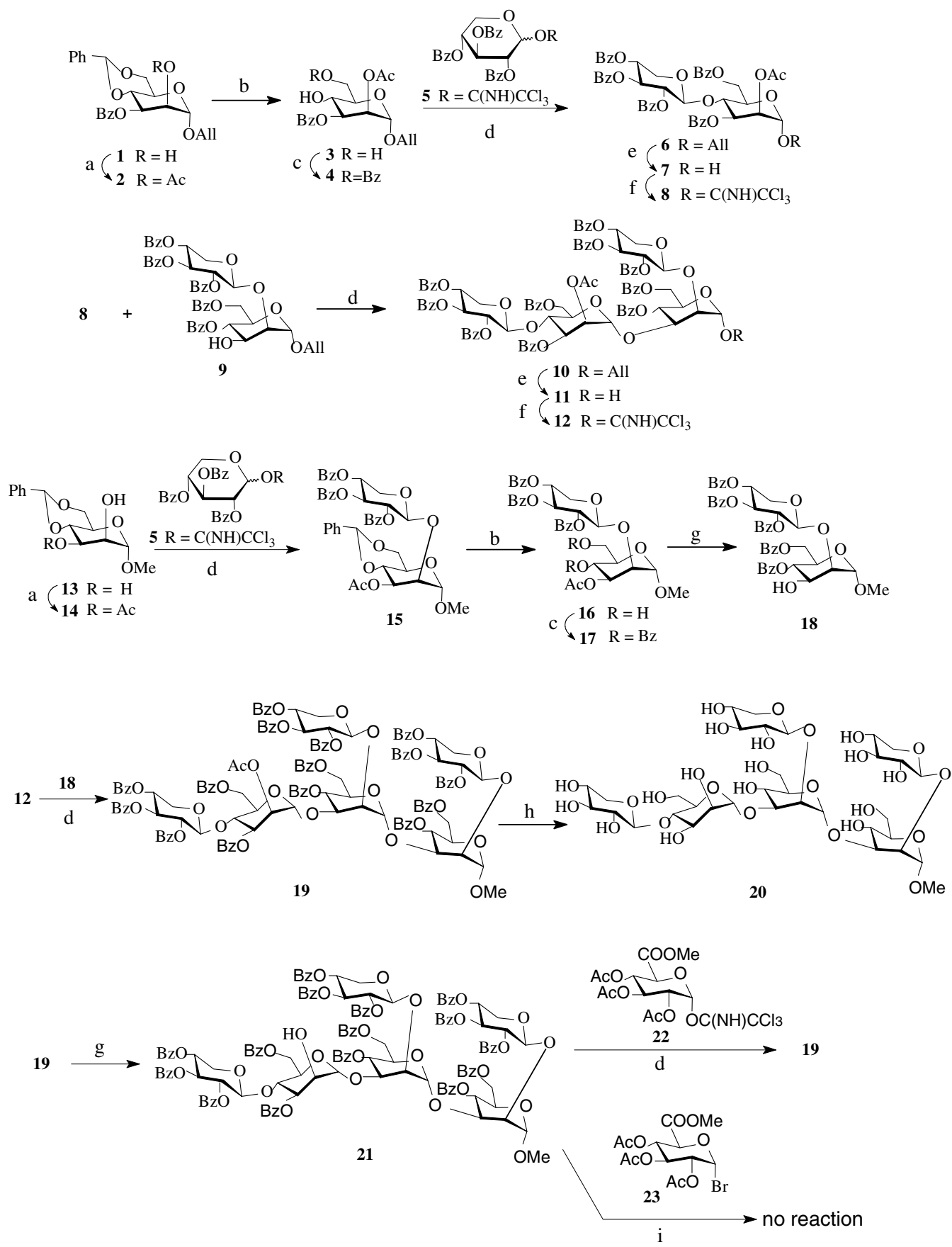

Scheme 1. Reagents and conditions: (a) $\mathrm{Ac}_{2} \mathrm{O}$, Pyridine $\left(\mathrm{CH}_{2} \mathrm{Cl}_{2}\right.$ ); (b) $90 \% \mathrm{HOAc}-\mathrm{H}_{2} \mathrm{O}$; (c) BzCl-Pyridine; (d) $\mathrm{TMSOTf} \mathrm{CH}_{2} \mathrm{Cl}{ }_{2},-10{ }^{\circ} \mathrm{C}$ to rt; (e) $\mathrm{PdCl}_{2}, \mathrm{CH}_{2} \mathrm{Cl}_{2}-\mathrm{MeOH}$, rt, $4 \mathrm{~h}$; (f) $\mathrm{CCl}_{3} \mathrm{CN}, \mathrm{K}_{2} \mathrm{CO}_{3}, \mathrm{CH}_{2} \mathrm{Cl}_{2}, 10 \mathrm{~h}$; (g) $4 \%(7 \%) \mathrm{CH}_{3} \mathrm{COCl}$ in $\mathrm{CH}_{2} \mathrm{Cl}_{2}-\mathrm{MeOH}, 0^{\circ} \mathrm{C}-\mathrm{rt}$; (h) satd NH${ }_{3}-\mathrm{MeOH}, \mathrm{rt}, 72 \mathrm{~h}$; (i) silver triflate, $\mathrm{CH}_{2} \mathrm{Cl}_{2}$, 2,4-lutidine.

\subsection{Allyl 2- $O$-acetyl-3- $O$-benzoyl-4,6- $O$-benzylidene- $\alpha$-D- mannopyranoside (2)}

Compound 1 ( $4.14 \mathrm{~g}, 10 \mathrm{mmol})$ was dissolved in pyridine $(30 \mathrm{~mL})$, and $\mathrm{Ac}_{2} \mathrm{O}(3.00 \mathrm{~mL}, 25 \mathrm{mmol})$ was added. The mixture was stirred at $\mathrm{rt}$ for $12 \mathrm{~h}$, then was concentrated to give a residue. Purification of the residue by silica gel column chromatography (3:1 petroleum ether-EtOAc) gave $2(4.96 \mathrm{~g}, 90.0 \%)$ as a syrup: $[\alpha]_{\mathrm{D}}+42.0$ (c 1.0,
$\left.\mathrm{CHCl}_{3}\right) ;{ }^{1} \mathrm{H}$ NMR $\left(400 \mathrm{MHz}, \mathrm{CDCl}_{3}\right): \delta 7.99-7.29(\mathrm{~m}$, $10 \mathrm{H}, 2 \mathrm{Ph} H), 5.88\left(\mathrm{~m}, 1 \mathrm{H}, \mathrm{CH}_{2}=\mathrm{CHCH}_{2} \mathrm{O}\right), 5.72(\mathrm{dd}$, $\left.1 \mathrm{H}, J_{2,3} 3.6 \mathrm{~Hz}, J_{3,4} 10.3 \mathrm{~Hz}, \mathrm{H}-3\right), 5.63(\mathrm{~s}, 1 \mathrm{H}, \mathrm{PhCHO}$ ), $5.50\left(\mathrm{dd}, \quad 1 \mathrm{H}, \quad J_{1,2} 1.3 \mathrm{~Hz}, \mathrm{H}-2\right), \quad 5.30(\mathrm{~m}, \quad 1 \mathrm{H}$, $\left.\mathrm{CH}_{2}=\mathrm{CHCH}_{2} \mathrm{O}\right), 5.20\left(\mathrm{~m}, 1 \mathrm{H}, \mathrm{CH}_{2}=\mathrm{CHCH}_{2} \mathrm{O}\right), 4.88$ (d, $\left.1 \mathrm{H}, J_{1,2} 1.3 \mathrm{~Hz}, \mathrm{H}-1\right), 4.23$ (dd, $1 \mathrm{H}, J 4.8,10.6 \mathrm{~Hz}, \mathrm{H}-$ 6a), $4.18\left(\mathrm{~m}, 1 \mathrm{H}, \mathrm{CH}_{2}=\mathrm{CHCH}_{2} \mathrm{O}\right), 4.08(\mathrm{dd}, 1 \mathrm{H}, J 10.0$, $10.6 \mathrm{~Hz}, \mathrm{H}-6 \mathrm{~b}), 4.02\left(\mathrm{~m}, 1 \mathrm{H}, \mathrm{CH}_{2}=\mathrm{CHCH}_{2} \mathrm{O}\right), 3.98$ (ddd, $1 \mathrm{H}, J 4.8,10.0,10.6 \mathrm{~Hz}, \mathrm{H}-5), 3.83$ (dd, $1 \mathrm{H}$, 
$\left.J_{3,4}=J_{4,5} 10.0 \mathrm{~Hz}, \mathrm{H}-4\right), 2.14$ (s, 3H, CH $\left.\mathrm{H}_{3} \mathrm{CO}\right)$. Anal. Calcd for $\mathrm{C}_{25} \mathrm{H}_{26} \mathrm{O}_{8}$ : C, 66.08; H, 5.73. Found: C, 65.94; H, 5.77 .

\subsection{Allyl 2-O-acetyl-3-O-benzoyl- $\alpha$-D-mannopyranoside} (3)

A mixture of $2(4.1 \mathrm{~g}, 9.1 \mathrm{mmol})$ and $90 \% \mathrm{HOAc}-\mathrm{H}_{2} \mathrm{O}$ $(80 \mathrm{~mL})$ was stirred for $2 \mathrm{~h}$ at $70^{\circ} \mathrm{C}$, then concentrated to dryness. Purification of the residue by silica gel column chromatography (1:1 petroleum ether-EtOAc) gave 3 $(2.66 \mathrm{~g}, 80 \%)$ as a syrup: $[\alpha]_{\mathrm{D}}+42.0\left(c 1.0, \mathrm{CHCl}_{3}\right) ;{ }^{1} \mathrm{H}$ NMR $\left(400 \mathrm{MHz}, \mathrm{CDCl}_{3}\right): \delta$ 7.98-7.41 (m, 5H, $\left.\mathrm{PhH}\right)$, $5.89\left(\mathrm{~m}, 1 \mathrm{H}, \mathrm{CH}_{2}=\mathrm{CHCH}_{2} \mathrm{O}\right), 5.47\left(\mathrm{dd}, 1 \mathrm{H}, J_{2,3} 3.5 \mathrm{~Hz}\right.$, $\left.J_{3,4} 9.9 \mathrm{~Hz}, \mathrm{H}-3\right), 5.37$ (dd, $\left.1 \mathrm{H}, J_{1,2} 1.6 \mathrm{~Hz}, \mathrm{H}-2\right), 5.30$ (m, $\left.1 \mathrm{H}, \mathrm{CH}_{2}=\mathrm{CHCH}_{2} \mathrm{O}\right), 5.20\left(\mathrm{~m}, 1 \mathrm{H}, \mathrm{CH}_{2}=\mathrm{CHCH}_{2} \mathrm{O}\right)$, $4.88(\mathrm{~d}, 1 \mathrm{H}, \mathrm{H}-1), 4.20\left(\mathrm{~m}, 1 \mathrm{H}, \mathrm{CH}_{2}=\mathrm{CHCH}_{2} \mathrm{O}\right), 4.15$ $\left(\mathrm{dd}, \quad 1 \mathrm{H}, \quad J_{3,4}=J_{4.5}=9.9 \mathrm{~Hz}, \mathrm{H}-4\right), 4.12 \quad(\mathrm{~m}, 1 \mathrm{H}$, $\left.\mathrm{CH}_{2}=\mathrm{CHCH}_{2} \mathrm{O}\right), 3.93-3.91$ (m, 2H, H-6a, H-6b), 3.81 $(\mathrm{m}, 1 \mathrm{H}, \mathrm{H}-5), 2.10\left(\mathrm{~s}, 3 \mathrm{H}, \mathrm{H}_{3} \mathrm{CO}\right)$. Anal. Calcd for $\mathrm{C}_{18} \mathrm{H}_{22} \mathrm{O}_{8}$ : C, 59.02; H, 6.01. Found: C, 59.32; H, 5.98.

\subsection{Allyl 2- $O$-acetyl-3,6-di- $O$-benzoyl- $\alpha-D-m a n n o p y r-$ anoside (4)}

Compound 3 (2.52 g, $7.0 \mathrm{mmol})$ was dissolved in anhyd $\mathrm{CH}_{2} \mathrm{Cl}_{2}(30 \mathrm{~mL})$ containing pyridine $(4.1 \mathrm{~mL}, 50 \mathrm{mmol})$, then under $\mathrm{N}_{2}$ protection and stirring, a solution of benzoyl chloride $(0.5 \mathrm{~mL}, 7.0 \mathrm{mmol})$ in anhyd $\mathrm{CH}_{2} \mathrm{Cl}_{2}$ $(6 \mathrm{~mL})$ was added dropwise within $30 \mathrm{~min}$ at $0^{\circ} \mathrm{C}$. The reaction temperature slowly raised to $\mathrm{rt}$. After stirring the mixture for $8 \mathrm{~h}$, TLC (3:1 petroleum ether-EtOAc) indicated that the reaction was complete. The reaction mixture was concentrated to give a residue. Purification of the residue by silica gel column chromatography (3:1 petroleum ether-EtOAc) gave $4(2.95 \mathrm{~g}, 90 \%)$ as a syrup: $[\alpha]_{\mathrm{D}}+46.1$ (c 1.0, $\left.\mathrm{CHCl}_{3}\right) ;{ }^{1} \mathrm{H}$ NMR $(400 \mathrm{MHz}$, $\left.\mathrm{CDCl}_{3}\right): \delta 8.10-7.39(\mathrm{~m}, 10 \mathrm{H}, 2 \mathrm{PhH}), 5.90(\mathrm{~m}, 1 \mathrm{H}$, $\left.\mathrm{CH}_{2}=\mathrm{CHCH}_{2} \mathrm{O}\right), 5.54\left(\mathrm{dd}, 1 \mathrm{H}, J_{2,3} 3.4 \mathrm{~Hz}, J_{3,4} 9.6 \mathrm{~Hz}\right.$, $\mathrm{H}-3), 5.38$ (dd, 1H, $\left.J_{1,2} 1.6 \mathrm{~Hz}, \mathrm{H}-2\right), 5.30(\mathrm{~m}, 1 \mathrm{H}$, $\left.\mathrm{CH}_{2}=\mathrm{CHCH}_{2} \mathrm{O}\right), 5.20\left(\mathrm{~m}, 1 \mathrm{H}, \mathrm{CH}_{2}=\mathrm{CHCH}_{2} \mathrm{O}\right), 4.92$ (d, 1H, H-1), 4.73-4.64 (m, 2H, H-6a, H-6b), 4.16 (m, $\left.1 \mathrm{H}, \mathrm{CH}_{2}=\mathrm{CHCH}_{2} \mathrm{O}\right), 4.12\left(\mathrm{dd}, 1 \mathrm{H}, J_{3,4}=J_{4,5}=9.6 \mathrm{~Hz}\right.$, $\mathrm{H}-4), 4.12\left(\mathrm{~m}, 1 \mathrm{H}, \mathrm{CH}_{2}=\mathrm{CHCH}_{2} \mathrm{O}\right), 4.08(\mathrm{~m}, 1 \mathrm{H}, \mathrm{H}-5)$, 2.10 (s, 3H, $\mathrm{H}_{3} \mathrm{CO}$ ). Anal. Calcd for $\mathrm{C}_{25} \mathrm{H}_{26} \mathrm{O}_{9}$ : C, 63.83; H, 5.53. Found: C, 63.99; H, 5.51.

\subsection{Allyl 2,3,4-tri- $O$-benzoyl- $\beta$-D-xylopyranosyl-( $\rightarrow 4)$ - 2- $O$-acetyl-3,6-di- $O$-benzoyl- $\alpha$-D-mannopyranoside (6)}

Compound $4(2.64 \mathrm{~g}, 5.60 \mathrm{mmol})$ and 2,3,4-tri- $O$-benzoyl- $\alpha$-D-xylopyranosyl trichloroacetimidate $5(3.62 \mathrm{~g}$, $6.0 \mathrm{mmol})$ were dried together under high vacuum for $2 \mathrm{~h}$, then dissolved in anhyd $\mathrm{CH}_{2} \mathrm{Cl}_{2}(50 \mathrm{~mL})$. TMSOTf $(10 \mu \mathrm{L}, 0.09 \mathrm{mmol})$ was added dropwise at $-10^{\circ} \mathrm{C}$ with nitrogen protection. The reaction mixture was stirred for
$3 \mathrm{~h}$, during which time the temperature was gradually warmed to ambient temperature. Then the mixture was neutralized with $\mathrm{Et}_{3} \mathrm{~N}$ and concentrated to dryness. Purification of the residue by silica gel column chromatography (3:1 petroleum ether-EtOAc) gave 6 $(4.80 \mathrm{~g}, 90 \%)$ as a foamy solid: $[\alpha]_{\mathrm{D}}-25.5$ (c 1.0, $\left.\mathrm{CHCl}_{3}\right) ;{ }^{1} \mathrm{H} \mathrm{NMR}\left(400 \mathrm{MHz}, \mathrm{CDCl}_{3}\right): \delta 8.10-7.17(\mathrm{~m}$, $25 \mathrm{H}, 5 \mathrm{Ph} H), 5.89\left(\mathrm{~m}, 1 \mathrm{H}, \mathrm{CH}_{2}=\mathrm{CHCH}_{2} \mathrm{O}\right), 5.76(\mathrm{dd}$, $1 \mathrm{H}, J_{2,3} 3.4 \mathrm{~Hz}, J_{3,4} 9.6 \mathrm{~Hz}, \mathrm{H}-3$ of $\left.\operatorname{Man} p\right), 5.66(\mathrm{dd}, 1 \mathrm{H}$, $J_{2,3}=J_{3,4}=7.1 \mathrm{~Hz}, \mathrm{H}-3$ of $\left.\mathrm{Xyl} p\right), 5.37$ (dd, $1 \mathrm{H}, J_{1,2}$ $1.7 \mathrm{~Hz}, \mathrm{H}-2$ of $\operatorname{Man} p), 5.32\left(\mathrm{dd}, 1 \mathrm{H}, J_{1,2} 5.2 \mathrm{~Hz}, \mathrm{H}-2\right.$ of $\mathrm{Xyl} p), \quad 5.30-5.10(\mathrm{~m}, 3 \mathrm{H}, \quad \mathrm{H}-4$ of $\mathrm{Man} p$, $\left.\mathrm{CH}_{2}=\mathrm{CHCH}_{2} \mathrm{O}\right), 4.97$ (d, 1H, $J_{1,2} 5.2 \mathrm{~Hz}, \mathrm{H}-1$ of Xylp), $4.87\left(\mathrm{~d}, 1 \mathrm{H}, J_{1,2} 1.7 \mathrm{~Hz}, \mathrm{H}-1\right.$ of $\left.\operatorname{Man} p\right), 4.63\left(\mathrm{dd}, 1 \mathrm{H}, J_{5,6 \mathrm{a}}\right.$ $1.8, J_{6 \mathrm{a}, 6 \mathrm{~b}} 12.2 \mathrm{~Hz}, \mathrm{H}-6 \mathrm{a}$ of $\left.\operatorname{Man} p\right), 4.48\left(\mathrm{dd}, 1 \mathrm{H}, J_{5,6 \mathrm{~b}} 4.2\right.$, $J_{6 \mathrm{a}, 6 \mathrm{~b}} 12.2 \mathrm{~Hz}, \quad \mathrm{H}-6 \mathrm{~b}$ of $\left.\operatorname{Man} p\right), 4.35(\mathrm{dd}, 1 \mathrm{H}$, $J_{3,4}=J_{4,5}=9.6 \mathrm{~Hz}, \mathrm{H}-4$ of $\left.\operatorname{Man} p\right), 4.10\left(\mathrm{dd}, 1 \mathrm{H}, J_{4,5 \mathrm{a}}\right.$ $3.0 \mathrm{~Hz}, J_{5 \mathrm{a}, 5 \mathrm{~b}} 11.8 \mathrm{~Hz}, \mathrm{H}-5 \mathrm{a}$ of Xylp), 4.17-3.26 (m, 4H), 2.07 (s, 3H, $\left.\mathrm{H}_{3} \mathrm{CO}\right)$. Anal. Calcd for $\mathrm{C}_{51} \mathrm{H}_{46} \mathrm{O}_{16}: \mathrm{C}$, 66.96; H, 5.03. Found: C, 67.07; H, 5.22.

3.6. 2,3,4-Tri- $O$-benzoyl- $\beta$-D-xylopyranosyl-( $1 \rightarrow 4)-2-O$ acetyl-3,6-di- $O$-benzoyl-D-mannopyranose (7)

To a solution of $6(4.21 \mathrm{~g}, 4.6 \mathrm{mmol})$ in anhyd $\mathrm{CH}_{2} \mathrm{Cl}_{2}$ $(10 \mathrm{~mL})$ and anhyd $\mathrm{MeOH}(40 \mathrm{~mL}), \mathrm{PdCl}_{2}$ (450 mg, $2.55 \mathrm{mmol}$ ) was added with $\mathrm{N}_{2}$ protection. After stirring the reaction mixture for $4 \mathrm{~h}$ at $\mathrm{rt}$, TLC (2:1 petroleum ether-EtOAc) indicated that the reaction was complete. Then the mixture was filtered, and the solution was concentrated to dryness. Purification of the residue by column chromatography (2:1 petroleum ether-EtOAc) gave $7(3.62 \mathrm{~g}, 85 \%)$ as a syrup, and the $\alpha$-anomer was isolated and characterized: $[\alpha]_{\mathrm{D}}-45.6\left(c 1.0, \mathrm{CHCl}_{3}\right) ;{ }^{1} \mathrm{H}$ NMR (400 MHz, $\left.\mathrm{CDCl}_{3}\right): \delta 8.10-7.17(\mathrm{~m}, 25 \mathrm{H}, 5 \mathrm{PhH})$, $5.80\left(\mathrm{dd}, 1 \mathrm{H}, J_{2,3} 3.3 \mathrm{~Hz}, J_{3,4} 9.6 \mathrm{~Hz}, \mathrm{H}-3\right.$ of Manp), 5.66 $\left(\mathrm{dd}, 1 \mathrm{H}, J_{2,3}=J_{3,4}=7.1 \mathrm{~Hz}, \mathrm{H}-3\right.$ of Xylp), $5.38(\mathrm{dd}, 1 \mathrm{H}$, $J_{1,2}=1.6 \mathrm{~Hz}, \mathrm{H}-2$ of $\left.\operatorname{Man} p\right), 5.33\left(\mathrm{dd}, 1 \mathrm{H}, J_{1,2} 5.2 \mathrm{~Hz}, \mathrm{H}-\right.$ 2 of Xylp), $5.23\left(\mathrm{~d}, 1 \mathrm{H}, J_{1,2} 1.6 \mathrm{~Hz}, \mathrm{H}-1\right.$ of $\left.\operatorname{Man} p\right), 5.08$ $(\mathrm{m}, 1 \mathrm{H}, \mathrm{H}-4$ of $\mathrm{Xyl} p), 5.00\left(\mathrm{~d}, 1 \mathrm{H}, J_{1,2} 5.2 \mathrm{~Hz}, \mathrm{H}-1\right.$ of $\mathrm{Xyl} p), 4.66\left(\mathrm{dd}, 1 \mathrm{H}, J_{5,6 \mathrm{a}} 1.6 \mathrm{~Hz}, J_{6 \mathrm{a}, 6 \mathrm{~b}} 12.2 \mathrm{~Hz}, \mathrm{H}-6 \mathrm{a}\right.$ of $\operatorname{Man} p), 4.46\left(\mathrm{dd}, 1 \mathrm{H}, J_{5,6 \mathrm{~b}} 3.4 \mathrm{~Hz}, \mathrm{H}-6 \mathrm{~b}\right.$ of $\left.\operatorname{Man} p\right), 4.38$ $\left(\mathrm{dd}, 1 \mathrm{H}, J_{3,4}=J_{4,5}=9.6 \mathrm{~Hz}, \mathrm{H}-4\right.$ of $\left.\operatorname{Man} p\right), 4.24(\mathrm{~m}, 1 \mathrm{H}$, H-5 of $\operatorname{Man} p), 4.03\left(\mathrm{dd}, 1 \mathrm{H}, J_{4,5 \mathrm{a}} 4.0 \mathrm{~Hz}, J_{5 \mathrm{a}, 5 \mathrm{~b}} 12.3 \mathrm{~Hz}\right.$, H-5a of Xylp), $3.30\left(\mathrm{dd}, 1 \mathrm{H}, J_{4,5 \mathrm{~b}} 6.6 \mathrm{~Hz}, J_{5 \mathrm{a}, 5 \mathrm{~b}} 12.3 \mathrm{~Hz}\right.$, $\mathrm{H}-5 \mathrm{~b}$ of $\mathrm{Xyl} p), 2.03$ (s, 3H, $\left.\mathrm{CH}_{3} \mathrm{CO}\right)$. Anal. Calcd for $\mathrm{C}_{48} \mathrm{H}_{42} \mathrm{O}_{16}$ : C, 65.90; H, 4.80. Found: C, 66.01; H, 4.71.

3.7. 2,3,4-Tri- $O$-benzoyl- $\beta$-D-xylopyranosyl-( $1 \rightarrow 4)-2-O$ acetyl-3,6-di- $O$-benzoyl- $\alpha$-D-mannopyranosyl trichloroacetimidate (8)

Compound 7 (3.20 g, $3.7 \mathrm{mmol})$ was dissolved in $\mathrm{CH}_{2} \mathrm{Cl}_{2}$ $(40 \mathrm{~mL})$, and $\mathrm{CCl}_{3} \mathrm{CN}(0.5 \mathrm{~mL}, 5 \mathrm{mmol})$ and $\mathrm{K}_{2} \mathrm{CO}_{3}$ $(1.5 \mathrm{~g})$ were added. The reaction mixture was stirred for 
$10 \mathrm{~h}$, at the end of which time TLC (2:1 petroleum etherEtOAc) indicated that the reaction was complete. Then the mixture was filtered, and the solution was concentrated to dryness. Purification of the residue on a silica gel column with 3:1 petroleum ether-EtOAc furnished the disaccharide donor $8(3.50 \mathrm{~g}, 90 \%)$ as a foamy solid: $[\alpha]_{\mathrm{D}}-18.1\left(c 0.8, \mathrm{CHCl}_{3}\right) ;{ }^{1} \mathrm{H}$ NMR $\left(400 \mathrm{MHz}, \mathrm{CDCl}_{3}\right)$ $\delta 8.73(\mathrm{~s}, 1 \mathrm{H}, \mathrm{CNHCCl}), 8.08-7.16(\mathrm{~m}, 25 \mathrm{H}, 5 \mathrm{Ph} H)$, $6.28\left(\mathrm{~s}, 1 \mathrm{H}, J_{1,2} 2.0 \mathrm{~Hz}, \mathrm{H}-1\right.$ of $\left.\operatorname{Man} p\right), 5.80\left(\mathrm{dd}, 1 \mathrm{H}, J_{2,3}\right.$ $3.4 \mathrm{~Hz}, J_{3,4} 9.6 \mathrm{~Hz}, \mathrm{H}-3$ of $\left.\operatorname{Man} p\right), 5.65(\mathrm{dd}, 1 \mathrm{H}$, $J_{2,3}=J_{3,4}=6.6 \mathrm{~Hz}, \mathrm{H}-3$ of Xylp), $5.60(\mathrm{dd}, 1 \mathrm{H}, \mathrm{H}-2$ of $\operatorname{Man} p), 5.31\left(\mathrm{dd}, 1 \mathrm{H}, J_{1,2} 4.7 \mathrm{~Hz}, \mathrm{H}-2\right.$ of Xylp), 5.09 (m, $1 \mathrm{H}, \mathrm{H}-4$ of Xylp), $5.03\left(\mathrm{~d}, 1 \mathrm{H}, J_{1,2} 4.7 \mathrm{~Hz}, \mathrm{H}-1\right.$ of Xylp), $4.72\left(\mathrm{dd}, 1 \mathrm{H}, J_{5,6 \mathrm{a}} 1.9 \mathrm{~Hz}, J_{6 \mathrm{a}, 6 \mathrm{~b}} 12.4 \mathrm{~Hz}, \mathrm{H}-6 \mathrm{a}\right.$ of Man $p$ ), $4.53\left(\mathrm{dd}, 1 \mathrm{H}, J_{5,6 \mathrm{~b}} 3.8 \mathrm{~Hz}, \mathrm{H}-6 \mathrm{~b}\right.$ of Manp), $4.49(\mathrm{dd}, 1 \mathrm{H}$, $J_{3,4}=J_{4,5}=9.6 \mathrm{~Hz}, \mathrm{H}-4$ of Manp $), 4.22(\mathrm{~m}, 1 \mathrm{H}, \mathrm{H}-5$ of $\operatorname{Man} p), 4.10\left(\mathrm{dd}, 1 \mathrm{H}, J_{4,5 \mathrm{a}} 4.5 \mathrm{~Hz}, J_{5 \mathrm{a}, 5 \mathrm{~b}} 12.4 \mathrm{~Hz}, \mathrm{H}-5 \mathrm{a}\right.$ of Xylp), 3.33 (dd, $1 \mathrm{H}, J_{4,5 \mathrm{~b}} 6.0 \mathrm{~Hz}, J_{5 \mathrm{a}, 5 \mathrm{~b}} 12.4 \mathrm{~Hz}, \mathrm{H}-5 \mathrm{~b}$ of Xylp), $2.10\left(\mathrm{~s}, 3 \mathrm{H}, \mathrm{CH}_{3} \mathrm{CO}\right.$ ). Anal. Calcd for $\mathrm{C}_{50} \mathrm{H}_{42} \mathrm{Cl}_{3} \mathrm{NO}_{16}: \mathrm{C}, 58.88 ; \mathrm{H}, 4.12$. Found: $\mathrm{C}, 58.62 ; \mathrm{H}$, 4.21.

\subsection{Allyl 2,3,4-tri- $O$-benzoyl- $\beta$-D-xylopyranosyl-( $(\rightarrow 4)$ - 2- $O$-acetyl-3,6-di- $O$-benzoyl- $\alpha$-D-mannopyranosyl- $(1 \rightarrow 3)$-[2,3,4-tri- $O$-benzoyl- $\beta$-D-xylopyranosyl-( $(\rightarrow 2)]-$ 4,6-di- $O$-benzoyl- $\alpha$-D-mannopyranoside (10)}

Compound $8 \quad(2.64 \mathrm{~g}, \quad 2.60 \mathrm{mmol})$ and $9 \quad(2.27 \mathrm{~g}$, $2.60 \mathrm{mmol}$ ) were dried together under high vacuum for $2 \mathrm{~h}$, then dissolved in anhyd $\mathrm{CH}_{2} \mathrm{Cl}_{2}(50 \mathrm{~mL})$. TMSOTf $(15 \mu \mathrm{L}, 0.12 \mathrm{mmol})$ was added dropwise at $-10{ }^{\circ} \mathrm{C}$ with nitrogen protection. The reaction mixture was stirred for $3 \mathrm{~h}$, during which time it was allowed to warm to ambient temperature. The mixture was then neutralized with $\mathrm{Et}_{3} \mathrm{~N}$ and concentrated to dryness. Purification of the residue by silica gel column chromatography (2:1 petroleum ether-EtOAc) gave $\mathbf{1 0}$ $(3.36 \mathrm{~g}, 75 \%)$ as a foamy solid: $[\alpha]_{\mathrm{D}}-36.3$ (c 1.0, $\left.\mathrm{CHCl}_{3}\right) ;{ }^{1} \mathrm{H}$ NMR $\left(\mathrm{CDCl}_{3}, 400 \mathrm{MHz}\right): \delta 8.15-7.02(\mathrm{~m}$, $50 \mathrm{H}, 10 \mathrm{Ph} H), 5.85\left(\mathrm{dd}, 1 \mathrm{H}, J_{2,3} 3.3 \mathrm{~Hz}, J_{3,4} 8.9 \mathrm{~Hz}, \mathrm{H}-3\right.$ of Manp), $5.75\left(\mathrm{dd}, 1 \mathrm{H}, J_{2,3}=J_{3,4}=5.9 \mathrm{~Hz}, \mathrm{H}-3\right.$ of $\mathrm{Xyl}), 5.70\left(\mathrm{~m}, 1 \mathrm{H}, \mathrm{CH}_{2}=\mathrm{CHCH}_{2} \mathrm{O}\right), 5.65(\mathrm{dd}, 1 \mathrm{H}$, $J_{2,3}=J_{3,4}=7.2 \mathrm{~Hz}, \quad \mathrm{H}-3$ of Xylp), $5.61(\mathrm{dd}, 1 \mathrm{H}$, $J_{3,4}=J_{4,5}=10.0 \mathrm{~Hz}, \mathrm{H}-4$ of Manp), $5.40\left(\mathrm{dd}, 1 \mathrm{H}, J_{1,2}\right.$ $5.2 \mathrm{~Hz}, \mathrm{H}-2$ of Xylp), $5.30\left(\mathrm{dd}, 1 \mathrm{H}, J_{1,2} 5.1 \mathrm{~Hz}, \mathrm{H}-2\right.$ of $\mathrm{Xyl} p), 5.20(\mathrm{~m}, 1 \mathrm{H}, \mathrm{H}-4$ of Xylp), $5.18(\mathrm{~m}, 1 \mathrm{H}, \mathrm{H}-4$ of Xylp), 5.15-5.05 (m, 4H), $4.96\left(\mathrm{~d}, 1 \mathrm{H}, J_{1,2} 5.1 \mathrm{~Hz}, \mathrm{H}-1\right.$ of Xylp), 4.95 (d, 1H, $J_{1,2} 5.2 \mathrm{~Hz}, \mathrm{H}-1$ of Xylp), 4.87 (s, $1 \mathrm{H}, \mathrm{H}-1$ of Manp), 4.81-3.25 (m, 15H), $1.94(\mathrm{~s}, 3 \mathrm{H}$, $\left.\mathrm{COCH}_{3}\right) ;{ }^{13} \mathrm{C}$ NMR $\left(100 \mathrm{MHz}, \mathrm{CDCl}_{3}\right): 168.6$ (C $\left.\mathrm{OCH}_{3}\right), 165.9,165.7,165.4,165.4,165.3,165.1,164.9$, $164.9, \quad 164.8, \quad 164.6, \quad(10 \mathrm{C}, \quad 10 \quad \mathrm{COPh}), \quad 118.1$ $\left(\mathrm{OCH}_{2} \mathrm{CH}=C_{2}\right), 101.0,99.4,98.4,96.3\left(4 \mathrm{C}, \mathrm{C}-1^{\mathrm{I}-\mathrm{IV}}\right)$, $75.62,75.13,70.55,70.12,70.10,69.96,69.60,69.31$, $69.27,69.05,68.85,68.58,68.40,68.30,63.83,62.93$,
61.44, 60.25 (C-2 to C-6), $20.3\left(\mathrm{COCH}_{3}\right)$. Anal. Calcd for $\mathrm{C}_{99} \mathrm{H}_{84} \mathrm{O}_{30}$ : C, 67.36; $\mathrm{H}, 4.86$. Found: $\mathrm{C}, 67.49 ; \mathrm{H}$, 5.03 .

3.9. 2,3,4-Tri- $O$-benzoyl- $\beta$-D-xylopyranosyl-(1 $\rightarrow 4)$-2- $O$ acetyl-3,6-di- $O$-benzoyl- $\alpha$-D-mannopyranosyl- $(1 \rightarrow 3)$ $[2,3,4$-tri- $O$-benzoyl- $\beta$-D-xylopyranosyl- $(1 \rightarrow 2)]-4,6$-di$O$-benzoyl- $\alpha$-D-mannopyranose (11)

To a solution of $\mathbf{1 0}(3.20 \mathrm{~g}, 1.86 \mathrm{mmol})$ in anhyd $\mathrm{CH}_{2} \mathrm{Cl}_{2}$ $(10 \mathrm{~mL})$ and anhyd $\mathrm{MeOH}(40 \mathrm{~mL}), \mathrm{PdCl}_{2}(220 \mathrm{mg}$, $1.22 \mathrm{mmol}$ ) was added with nitrogen protection. After stirring the reaction mixture for $4 \mathrm{~h}$ at $\mathrm{rt}$, TLC (1:1 petroleum ether-EtOAc) indicated that the reaction was complete. Then the mixture was filtered, and the solution was concentrated to dryness. Purification of the residue by column chromatography (2:1 petroleum ether-EtOAc) gave $\mathbf{1 1}(2.66 \mathrm{~g}, 85 \%)$ as a foamy solid: $[\alpha]_{\mathrm{D}}-39.4\left(c 1.0, \mathrm{CHCl}_{3}\right) ;{ }^{1} \mathrm{H} \mathrm{NMR}\left(\mathrm{CDCl}_{3}, 400 \mathrm{MHz}\right)$ : $\delta$ 8.16-7.04 (m, 50H, $10 \mathrm{Ph} H), 5.86\left(\mathrm{dd}, 1 \mathrm{H}, J_{2,3}\right.$ $3.3 \mathrm{~Hz}, J_{3,4} 8.7 \mathrm{~Hz}, \mathrm{H}-3$ of Manp), $5.77(\mathrm{dd}, 1 \mathrm{H}$, $J_{2,3}=J_{3,4}=5.1 \mathrm{~Hz}, \mathrm{H}-3$ of Xylp), $5.69\left(\mathrm{dd}, 1 \mathrm{H}, J_{2,3}=\right.$ $J_{3,4}=7.1 \mathrm{~Hz}, \mathrm{H}-3$ of Xylp), $5.67\left(\mathrm{dd}, 1 \mathrm{H}, J_{3,4}=J_{4,5}=\right.$ $10.1 \mathrm{~Hz}, \mathrm{H}-4$ of Manp), $5.42\left(\mathrm{dd}, 1 \mathrm{H}, J_{1,2} 5.3 \mathrm{~Hz}, \mathrm{H}-2\right.$ of $\mathrm{Xyl} p), 5.30-5.24(\mathrm{~m}, 3 \mathrm{H}), 5.18(\mathrm{~m}, 1 \mathrm{H}, \mathrm{H}-4$ of Xylp), $5.14\left(\mathrm{dd}, 1 \mathrm{H}, \mathrm{H}-2\right.$ of Manp), 5.07 (d, $1 \mathrm{H}, J_{1,2} 1.9 \mathrm{~Hz}, \mathrm{H}-1$ of Manp), 4.98 (d, 1H, $J_{1,2} 4.8 \mathrm{~Hz}, \mathrm{H}-1$ of Xylp), 4.94 (d, $1 \mathrm{H}, J_{1,2} 5.3 \mathrm{~Hz}, \mathrm{H}-1$ of Xylp), 4.87-3.25 (m, 13H), 1.89 (s, $\left.3 \mathrm{H}, \mathrm{COCH}_{3}\right) ;{ }^{13} \mathrm{C}$ NMR (100 $\left.\mathrm{MHz}, \mathrm{CDCl}_{3}\right): 168.6$ $\left(\mathrm{COCH}_{3}\right), 166.1,165.7,165.5,165.2,165.1,165.0,164.9$, 164.9, 164.8, 164.6, (10C, $10 \mathrm{COPh}), 101.1,99.3,98.1$, $92.0\left(4 \mathrm{C}, \mathrm{C}-1^{\mathrm{I}-\mathrm{IV}}\right), 75.18,74.57,70.63,70.30,70.13$, $69.97,69.64,69.57,69.27,69.16,68.81,68.45,68.30$, 63.89, 63.02, 61.57, 60.19 (C-2 to C-6), $20.3\left(\mathrm{COCH}_{3}\right)$. Anal. Calcd for $\mathrm{C}_{94} \mathrm{H}_{80} \mathrm{O}_{30}$ : C, 66.82; H, 4.74. Found: C, 66.93; H, 4.64.

\subsection{2,3,4-Tri- $O$-benzoyl- $\beta$-D-xylopyranosyl-(1 $\rightarrow 4)-2$ - $O$-acetyl-3,6-di- $O$-benzoyl- $\alpha$-D-mannopyranosyl-( $1 \rightarrow 3$ )- [2,3,4-tri- $O$-benzoyl- $\beta$-D-xylopyranosyl-( $(\rightarrow 2)]-4,6$-di- $O$-benzoyl- $\alpha$-D-mannopyranosyl trichloroacetimidate (12)}

Compound $11(2.6 \mathrm{~g}, 1.54 \mathrm{mmol})$ was dissolved in $\mathrm{CH}_{2} \mathrm{Cl}_{2}(30 \mathrm{~mL})$, and $\mathrm{CCl}_{3} \mathrm{CN}(0.5 \mathrm{~mL}, 5 \mathrm{mmol})$ and $\mathrm{K}_{2} \mathrm{CO}_{3}(1.0 \mathrm{~g})$ were added. The reaction mixture was stirred for $10 \mathrm{~h}$, at the end of which time TLC $(2: 1$ petroleum ether-EtOAc) indicated that the reaction was complete. Then the mixture was filtered, and the solution was concentrated to dryness. Purification of the residue on a silica gel column with 3:1 petroleum etherEtOAc as the eluent furnished the tetrasaccharide donor $12(2.54 \mathrm{~g}, 90.6 \%)$ as a foamy solid: $[\alpha]_{\mathrm{D}}-46.9$ (c 0.5 , $\left.\mathrm{CHCl}_{3}\right) ;{ }^{1} \mathrm{H}$ NMR $\left(\mathrm{CDCl}_{3}, 400 \mathrm{MHz}\right): \delta 8.71(\mathrm{~s}, 1 \mathrm{H}$, $\left.\mathrm{CNHCCl}_{3}\right), 8.15-7.02(\mathrm{~m}, 50 \mathrm{H}, 10 \mathrm{Ph} H), 6.38\left(\mathrm{~d}, 1 \mathrm{H}, J_{1,2}\right.$ $1.4 \mathrm{~Hz}, \mathrm{H}-1$ of $\operatorname{Man} p), 5.88\left(\mathrm{dd}, 1 \mathrm{H}, J_{2,3} 3.2 \mathrm{~Hz}, J_{3,4}\right.$ $8.9 \mathrm{~Hz}, \mathrm{H}-3$ of Manp), $5.85\left(\mathrm{dd}, 1 \mathrm{H}, J_{2,3}=J_{3,4}=5.0 \mathrm{~Hz}\right.$, 
H-3 of Xylp), $5.76\left(\mathrm{dd}, 1 \mathrm{H}, J_{3,4}=J_{4,5}=10.1 \mathrm{~Hz}, \mathrm{H}-4\right.$ of $\operatorname{Man} p), 5.66\left(\mathrm{dd}, 1 \mathrm{H}, J_{2,3}=J_{3,4}=7.1 \mathrm{~Hz}, \mathrm{H}-3\right.$ of Xylp), 5.43-5.40 (m, 2H), $5.30(\mathrm{~m}, 1 \mathrm{H}, \mathrm{H}-4$ of Xylp), 5.21-5.14 $(\mathrm{m}, 2 \mathrm{H}), 5.14\left(\mathrm{~d}, 1 \mathrm{H}, J_{1,2} 5.0 \mathrm{~Hz}, \mathrm{H}-1\right.$ of Xylp), $5.11(\mathrm{~s}$, $1 \mathrm{H}, \mathrm{H}-1$ of Manp), $4.97\left(\mathrm{~d}, 1 \mathrm{H}, J_{1,2} 5.2 \mathrm{~Hz}, \mathrm{H}-1\right.$ of Xylp), 4.87-3.30 (m, 13H), $2.03\left(\mathrm{~s}, 3 \mathrm{H}, \mathrm{COCH}_{3}\right) ;{ }^{13} \mathrm{C} \mathrm{NMR}$ $\left(100 \mathrm{MHz}, \mathrm{CDCl}_{3}\right): 168.6\left(\mathrm{COCH}_{3}\right), 165.9,165.6,165.4$, $165.3,165.2,165.1,165.0,164.9,164.8,164.6,(10 \mathrm{C}$, $10 \mathrm{COPh}), 101.1,99.5,98.5,94.8\left(4 \mathrm{C}, \mathrm{C}-1^{\mathrm{I}-\mathrm{IV}}\right), 90.5$ $\left(-\mathrm{CCl}_{3}\right), 75.16,74.99,74.77,71.65,70.70,70.31,69.88$, $69.60,69.33,69.15,69.00,68.43,68.40,63.30,62.59$, $61.60,60.64,60.31$ (C-2 to $\mathrm{C}-6), 20.2\left(\mathrm{COCH}_{3}\right)$. Anal. Calcd for $\mathrm{C}_{96} \mathrm{H}_{80} \mathrm{Cl}_{3} \mathrm{NO}_{30}$ : C, 62.85; H, 4.36. Found: C, 63.08; H, 4.54.

\subsection{Methyl 3- $O$-acetyl-4,6- $O$-benzylidene- $\alpha$-D-manno- pyranoside (14)}

Compound $13(2.90 \mathrm{~g}, 10 \mathrm{mmol})$ was dissolved in anhyd $\mathrm{CH}_{2} \mathrm{Cl}_{2} \quad(30 \mathrm{~mL})$ containing pyridine $(8.1 \mathrm{~mL}$, $100 \mathrm{mmol})$, a solution of $\mathrm{Ac}_{2} \mathrm{O}(1.20 \mathrm{~mL}, 10 \mathrm{mmol})$ in anhyd $\mathrm{CH}_{2} \mathrm{Cl}_{2}(10 \mathrm{~mL})$ was added dropwise within $30 \mathrm{~min}$ at $0{ }^{\circ} \mathrm{C}$. The reaction temperature was slowly raised to rt. After stirring the mixture for $12 \mathrm{~h}$, TLC (3:1 petroleum ether-EtOAc) indicated that the reaction was complete. The reaction mixture was concentrated to give a residue, and purification of the residue by column chromatography on a silica gel column (3:1 petroleum ether-EtOAc) gave compound $\mathbf{1 4}(2.62 \mathrm{~g}, 80.6 \%)$ as a syrup: $[\alpha]_{\mathrm{D}}+62.8\left(c\right.$ 1.0, $\left.\mathrm{CHCl}_{3}\right) ;{ }^{1} \mathrm{H}$ NMR $(400 \mathrm{MHz}$, $\left.\mathrm{CDCl}_{3}\right): \delta 7.46-7.26(\mathrm{~m}, 5 \mathrm{H}, \mathrm{Ph} H), 5.55(\mathrm{~s}, 1 \mathrm{H}$, $\left.\mathrm{PhCHO}_{2}\right), 5.32\left(\mathrm{dd}, 1 \mathrm{H}, J_{2,3} 3.3 \mathrm{~Hz}, J_{3,4} 10.1 \mathrm{~Hz}, \mathrm{H}-3\right)$, $4.75\left(\mathrm{~d}, 1 \mathrm{H}, J_{1,2} 1.5 \mathrm{~Hz}, \mathrm{H}-1\right), 4.30(\mathrm{dd}, 1 \mathrm{H}, J 4.2 \mathrm{~Hz}$, $10.1 \mathrm{~Hz}, \mathrm{H}-6 \mathrm{a}), 4.15$ (dd, $1 \mathrm{H}, J_{1,2} 1.5 \mathrm{~Hz}, J_{2.3} 3.3 \mathrm{~Hz}, \mathrm{H}-$ 2), 4.09 (dd, 1H, $J 10.1 \mathrm{~Hz}, 10.1 \mathrm{~Hz}, \mathrm{H}-6 \mathrm{~b}), 3.93$ (m, 1H, $\mathrm{H}-5), 3.84\left(\mathrm{dd}, 1 \mathrm{H}, J_{3,4}=J_{4,5}=10.1 \mathrm{~Hz}, \mathrm{H}-4\right), 3.40(\mathrm{~s}$, $\left.3 \mathrm{H}, \mathrm{OCH}_{3}\right), 2.13\left(\mathrm{~s}, 3 \mathrm{H}, \mathrm{CH}_{3} \mathrm{CO}\right)$. Anal. Calcd for $\mathrm{C}_{16} \mathrm{H}_{20} \mathrm{O}_{7}$ : C, 59.26; H, 6.17. Found: C, 59.42; H, 6.21.

\subsection{Methyl 2,3,4-tri- $O$-benzoyl- $\beta$-D-xylopyranosyl- $(1 \rightarrow 2)-3-O$-acetyl-4,6- $O$-benzylidene- $\alpha$-D-mannopyrano- side (15)}

Compound $14(2.11 \mathrm{~g}, 6.50 \mathrm{mmol})$ and 2,3,4-tri- $O$-benzoyl-D-xylopyranosyl trichloroacetimidate (5) $(4.00 \mathrm{~g}$, $6.60 \mathrm{mmol}$ ) were dried together under high vacuum for $2 \mathrm{~h}$, then dissolved in anhyd $\mathrm{CH}_{2} \mathrm{Cl}_{2}(50 \mathrm{~mL})$. TMSOTf $(15 \mu \mathrm{L}, 0.10 \mathrm{mmol})$ was added dropwise at $-10^{\circ} \mathrm{C}$ with $\mathrm{N}_{2}$ protection. The reaction mixture was stirred for $3 \mathrm{~h}$, during which time it was allowed to warm to ambient temperature. The mixture was then neutralized with $\mathrm{Et}_{3} \mathrm{~N}$ and concentrated to dryness. Purification of the residue by silica gel column chromatography (3:1 petroleum ether-EtOAc) gave $15(4.05 \mathrm{~g}, 80 \%)$ as a foamy solid: $[\alpha]_{\mathrm{D}}-38.9$ (c 1.0, $\left.\mathrm{CHCl}_{3}\right) ;{ }^{1} \mathrm{H} \mathrm{NMR}$ $\left(400 \mathrm{MHz}, \mathrm{CDCl}_{3}\right): \delta 8.15-7.33(\mathrm{~m}, 20 \mathrm{H}, 4 \mathrm{Ph} H), 5.70$ $\left(\mathrm{dd}, 1 \mathrm{H}, J_{2,3}=J_{3,4}=5.6 \mathrm{~Hz}, \mathrm{H}-3\right.$ of Xylp), $5.40(\mathrm{dd}, 1 \mathrm{H}$, $J_{1,2} 4.6 \mathrm{~Hz}, J_{2,3} 5.6 \mathrm{~Hz}, \mathrm{H}-2$ of Xylp), $5.31\left(\mathrm{dd}, 1 \mathrm{H}, J_{2,3}\right.$ $3.4 \mathrm{~Hz}, J_{3,4} 10.5 \mathrm{~Hz}, \mathrm{H}-3$ of Manp), $5.29(\mathrm{~m}, 1 \mathrm{H}, \mathrm{H}-4$ of $\mathrm{Xyl} p), 5.23\left(\mathrm{~s}, 1 \mathrm{H}, \mathrm{PhCHO}_{2}\right), 4.93\left(\mathrm{~d}, 1 \mathrm{H}, J_{1,2} 4.6 \mathrm{~Hz}, \mathrm{H}-\right.$ 1 of Xylp), $4.67\left(\mathrm{~d}, 1 \mathrm{H}, J_{1,2} 1.2 \mathrm{~Hz}, \mathrm{H}-1\right.$ of Manp), 4.60 (dd, $1 \mathrm{H}, J_{5,6 \mathrm{a}} 3.3 \mathrm{~Hz}, J_{6 \mathrm{a}, 6 \mathrm{~b}} 12.4 \mathrm{~Hz}, \mathrm{H}-6 \mathrm{a}$ of Manp), 4.28 (dd, $1 \mathrm{H}, J_{1,2} 1.2 \mathrm{~Hz}, J_{2,3} 3.4 \mathrm{~Hz}, \mathrm{H}-2$ of Manp), 4.08 (dd, $1 \mathrm{H}, J_{4,5 \mathrm{a}} 4.6 \mathrm{~Hz}, J_{5 \mathrm{a}, 5 \mathrm{~b}} 10.1 \mathrm{~Hz}, \mathrm{H}-5 \mathrm{a}$ of Xyl $\left.p\right), 3.98$ (dd, $1 \mathrm{H}, J_{4,5 \mathrm{~b}}=J_{5 \mathrm{a}, 5 \mathrm{~b}} 10.1 \mathrm{~Hz}, \mathrm{H}-5 \mathrm{~b}$ of Xylp), 3.82-3.76 (m, $2 \mathrm{H}, \mathrm{H}-5$ of $\operatorname{Man} p, \mathrm{H}-6 \mathrm{~b}$ of $\operatorname{Man} p), 3.48(\mathrm{dd}, 1 \mathrm{H}$, $J_{3,4}=J_{4,5}=10.1 \mathrm{~Hz}, \mathrm{H}-4$ of Manp), $3.32\left(\mathrm{~s}, 3 \mathrm{H}, \mathrm{CH}_{3} \mathrm{O}\right)$, $2.14\left(\mathrm{~s}, 3 \mathrm{H}, \mathrm{CH}_{3} \mathrm{CO}\right)$. Anal. Calcd for $\mathrm{C}_{42} \mathrm{H}_{40} \mathrm{O}_{14}: \mathrm{C}$, 65.63; H, 5.21. Found: C, 65.30; H, 5.38.

\subsection{Methyl 2,3,4-tri- $O$-benzoyl- $\beta$-D-xylopyranosyl- $(1 \rightarrow 2)$-3- $O$-acetyl- $\alpha$-D-mannopyranoside (16)}

A mixture of $\mathbf{1 5}(3.85 \mathrm{~g}, 5.0 \mathrm{mmol})$ and $90 \% \mathrm{HOAc}-\mathrm{H}_{2} \mathrm{O}$ $(60 \mathrm{~mL})$ was stirred for $2 \mathrm{~h}$ at $70^{\circ} \mathrm{C}$. The solution was concentrated to dryness. Purification of the residue by silica gel column chromatography (1:1 petroleum etherEtOAc) gave $16(2.71 \mathrm{~g}, 80 \%)$ as a foamy solid: $[\alpha]_{\mathrm{D}}-31.4$ ( c 1.0, $\left.\mathrm{CHCl}_{3}\right) ;{ }^{1} \mathrm{H}$ NMR $\left(400 \mathrm{MHz}, \mathrm{CDCl}_{3}\right)$ : $\delta 7.94-7.31(\mathrm{~m}, 15 \mathrm{H}, 3 \mathrm{Ph} H), 5.73\left(\mathrm{dd}, 1 \mathrm{H}, J_{2,3}=J_{3,4}=\right.$ $7.6 \mathrm{~Hz}, \mathrm{H}-3$ of Xylp), $5.38\left(\mathrm{dd}, 1 \mathrm{H}, J_{1,2} 5.6 \mathrm{~Hz}, J_{2,3}\right.$ $7.6 \mathrm{~Hz}, \mathrm{H}-2$ of $\mathrm{Xyl} p), 5.28(\mathrm{~m}, 1 \mathrm{H}, \mathrm{H}-4$ of $\mathrm{Xyl} p), 4.96$ (dd, $1 \mathrm{H}, J_{2,3} 3.2 \mathrm{~Hz}, J_{3,4} 10.0 \mathrm{~Hz}, \mathrm{H}-3$ of Manp), 4.72 (d, $1 \mathrm{H}, J_{1,2} 5.6 \mathrm{~Hz}, \mathrm{H}-1$ of Xylp), $4.47\left(\mathrm{~d}, 1 \mathrm{H}, J_{1,2} 1.6 \mathrm{~Hz}, \mathrm{H}-\right.$ 1 of Manp), $4.42\left(\mathrm{dd}, 1 \mathrm{H}, J_{4,5 \mathrm{a}} 4.4 \mathrm{~Hz}, J_{5 \mathrm{a}, 5 \mathrm{~b}} 12.0 \mathrm{~Hz}, \mathrm{H}-\right.$ 5 a of Xylp), $4.10\left(\mathrm{dd}, 1 \mathrm{H}, J_{1,2} 1.6 \mathrm{~Hz}, J_{2,3} 3.2 \mathrm{~Hz}, \mathrm{H}-2\right.$ of $\operatorname{Man} p), 4.01\left(\mathrm{dd}, 1 \mathrm{H}, J_{3,4}=J_{4,5}=10.0 \mathrm{~Hz}, \mathrm{H}-4\right.$ of Manp), 3.62-3.44 (m, 4H, H-5b of Xylp, H-6a of Manp, H-6b of Manp, H-5 of Manp), 3.16 (s, 3H, $\mathrm{CH}_{3} \mathrm{O}$ ), 2.10 (s, $3 \mathrm{H}, \mathrm{CH}_{3} \mathrm{CO}$ ). Anal. Calcd for $\mathrm{C}_{35} \mathrm{H}_{36} \mathrm{O}_{14}: \mathrm{C}, 61.76 ; \mathrm{H}$, 5.29. Found: C, 61.94; H, 5.38.

\subsection{Methyl 2,3,4-tri- $O$-benzoyl- $\beta$-D-xylopyranosyl- $(1 \rightarrow 2)-3-O$-acetyl-4,6-di- $O$-benzoyl- $\alpha$-D-mannopyrano- side (17)}

Compound 16 (2.5 g, $3.67 \mathrm{mmol})$ was dissolved in pyridine $(30 \mathrm{~mL})$, and benzoyl chloride $(2.47 \mathrm{~mL}, 20 \mathrm{mmol})$ was added. The mixture was stirred at $\mathrm{rt}$ for $12 \mathrm{~h}$, then quenched with $\mathrm{MeOH}(3 \mathrm{~mL})$. The reaction mixture was concentrated to give a residue. Purification of the residue by silica gel column chromatography (3:1 petroleum ether-EtOAc) gave $17(2.78 \mathrm{~g}, 85 \%)$ as a foamy solid: $[\alpha]_{\mathrm{D}}-27.5\left(c 1.0, \mathrm{CHCl}_{3}\right) ;{ }^{1} \mathrm{H}$ NMR $\left(400 \mathrm{MHz}, \mathrm{CDCl}_{3}\right)$ : $\delta$ 8.16-7.30 (m, 25H, $5 \mathrm{Ph} H), 5.70\left(\mathrm{dd}, 1 \mathrm{H}, J_{2,3}=\right.$ $J_{3,4}=5.1 \mathrm{~Hz}, \mathrm{H}-3$ of Xylp), $5.67\left(\mathrm{dd}, 1 \mathrm{H}, J_{3,4}=J_{4,5}=\right.$ $10.0 \mathrm{~Hz}, \mathrm{H}-4$ of Manp), $5.48\left(\mathrm{dd}, 1 \mathrm{H}, J_{2,3} 3.3 \mathrm{~Hz}, J_{3,4}\right.$ $10.0 \mathrm{~Hz}, \mathrm{H}-3$ of Manp), $5.37\left(\mathrm{dd}, 1 \mathrm{H}, J_{1,2} 5.0 \mathrm{~Hz}, J_{2,3}\right.$ $5.1 \mathrm{~Hz}, \mathrm{H}-2$ of Xylp), 5.27 (m, 1H, H-4 of Xylp), 4.99 (d, $1 \mathrm{H}, J_{1,2} 5.0 \mathrm{~Hz}, \mathrm{H}-1$ of Xylp), $4.81\left(\mathrm{~d}, 1 \mathrm{H}, J_{1,2} 1.4 \mathrm{~Hz}\right.$, $\mathrm{H}-1$ of Manp), $4.64\left(\mathrm{dd}, 1 \mathrm{H}, J_{4,5 \mathrm{a}} 3.0, J_{5 \mathrm{a}, 5 \mathrm{~b}} 12.4 \mathrm{~Hz}, \mathrm{H}-5 \mathrm{a}\right.$ of Xylp), $4.34\left(\mathrm{dd}, 1 \mathrm{H}, J_{5,6 \mathrm{a}} 3.0 \mathrm{~Hz}, J_{6 \mathrm{a}, 6 \mathrm{~b}} 11.8 \mathrm{~Hz}, \mathrm{H}-6 \mathrm{a}\right.$ 
of $\operatorname{Man} p), 4.24(\mathrm{dd}, 1 \mathrm{H}, \mathrm{H}-2$ of $\operatorname{Man} p), 4.18(\mathrm{~m}, 1 \mathrm{H}$, H-5 of Manp), $4.03\left(\mathrm{dd}, 1 \mathrm{H}, J_{5,6 \mathrm{~b}} 6.5 \mathrm{~Hz}, J_{6 \mathrm{a}, 6 \mathrm{~b}} 11.8 \mathrm{~Hz}\right.$, H-6b of Manp), 3.80 (dd, $1 \mathrm{H}, J_{4,5 \mathrm{~b}} 4.5 \mathrm{~Hz}, J_{5 \mathrm{a}, 5 \mathrm{~b}} 12.4 \mathrm{~Hz}$, $\mathrm{H}-5 \mathrm{~b}$ of $\mathrm{Xyl} p), 3.39$ (s, 3H, $\left.\mathrm{CH}_{3} \mathrm{O}\right), 1.98$ (s, 3H, $\mathrm{CH}_{3} \mathrm{CO}$ ). Anal. Calcd for $\mathrm{C}_{49} \mathrm{H}_{44} \mathrm{O}_{16}: \mathrm{C}, 66.22 ; \mathrm{H}, 4.95$. Found: C, 66.09; H, 5.01.

\subsection{Methyl 2,3,4-tri- $O$-benzoyl- $\beta$-D-xylopyranosyl- $(1 \rightarrow 2)-4,6$-di- $O$-benzoyl- $\alpha$-D-mannopyranoside (18)}

To a solution of $\mathbf{1 7}(2.71 \mathrm{~g}, 3.1 \mathrm{mmol})$ in anhyd $\mathrm{CH}_{2} \mathrm{Cl}_{2}$ $(10 \mathrm{~mL})$ was added anhyd $\mathrm{MeOH}(40 \mathrm{~mL})$, then $\mathrm{AcCl}$ $(2.0 \mathrm{~mL})$ was added to the reaction mixture at $0{ }^{\circ} \mathrm{C}$. The solution was stoppered in a flask and stirred at $\mathrm{rt}$ overnight, TLC (2:1 petroleum ether-EtOAc) showed that the starting material disappeared. The solution was neutralized with $\mathrm{Et}_{3} \mathrm{~N}$, then concentrated to dryness. Purification of the residue by silica gel column chromatography (2:1 petroleum ether-EtOAc) gave 18 $(2.20 \mathrm{~g}, 85 \%)$ as a foamy solid: $[\alpha]_{\mathrm{D}}-10.5$ (c 1.0, $\left.\mathrm{CHCl}_{3}\right) ;{ }^{1} \mathrm{H}$ NMR $\left(400 \mathrm{MHz}, \mathrm{CDCl}_{3}\right): \delta 8.04-7.30(\mathrm{~m}$, $25 \mathrm{H}, 5 \mathrm{PhH}), 5.77\left(\mathrm{dd}, 1 \mathrm{H}, J_{2,3}=J_{3,4}=6.8 \mathrm{~Hz}, \mathrm{H}-3\right.$ of $\mathrm{Xyl} p), 5.46\left(\mathrm{dd}, 1 \mathrm{H}, J_{3,4}=J_{4,5}=9.8 \mathrm{~Hz}, \mathrm{H}-4\right.$ of $\left.\operatorname{Man} p\right)$, $5.41\left(\mathrm{dd}, 1 \mathrm{H}, J_{1,2} 5.0 \mathrm{~Hz}, J_{2,3} 6.7 \mathrm{~Hz}, \mathrm{H}-2\right.$ of Xylp), 5.30 $(\mathrm{m}, 1 \mathrm{H}, \mathrm{H}-4$ of $\mathrm{Xyl} p), 5.01\left(\mathrm{~d}, 1 \mathrm{H}, J_{1,2} 5.0 \mathrm{~Hz}, \mathrm{H}-1\right.$ of $\mathrm{Xyl} p), 4.72\left(\mathrm{~d}, 1 \mathrm{H}, J_{1,2} 1.0 \mathrm{~Hz}, \mathrm{H}-1\right.$ of $\left.\operatorname{Man} p\right), 4.62(\mathrm{dd}$, $1 \mathrm{H}, J_{4,5 \mathrm{a}} 4.0 \mathrm{~Hz}, J_{5 \mathrm{a}, 5 \mathrm{~b}} 12.3 \mathrm{~Hz}, \mathrm{H}-5 \mathrm{a}$ of Xylp), 4.49 (dd, $1 \mathrm{H}, J_{5,6 \mathrm{a}} 2.6, J_{6 \mathrm{a}, 6 \mathrm{~b}} 11.9 \mathrm{~Hz}, \mathrm{H}-6 \mathrm{a}$ of $\left.\operatorname{Man} p\right), 4.24(\mathrm{dd}, 1 \mathrm{H}$, $J_{5,6 \mathrm{~b}} 5.8, J_{6 \mathrm{a}, 6 \mathrm{~b}} 11.9 \mathrm{~Hz}, \mathrm{H}-6 \mathrm{~b}$ of $\left.\mathrm{Man} p\right), 4.16-4.09(\mathrm{~m}$, $3 \mathrm{H}, \mathrm{H}-3$ of $\mathrm{Man} p, \mathrm{H}-5$ of Manp, H-2 of Manp), 3.87 $\left(\mathrm{dd}, 1 \mathrm{H}, J_{4,5 \mathrm{~b}} 6.5 \mathrm{~Hz}, J_{5 \mathrm{a}, 5 \mathrm{~b}} 12.3 \mathrm{~Hz}, \mathrm{H}-5 \mathrm{~b}\right.$ of Xylp), 1.60 (br s, $1 \mathrm{H}, \mathrm{OH}$ ). Anal. Calcd for $\mathrm{C}_{47} \mathrm{H}_{42} \mathrm{O}_{15}$ : C, 66.67; H, 5.20. Found: C, 66.89; H, 5.11.

\subsection{Methyl 2,3,4-tri- $O$-benzoyl- $\beta$-D-xylopyranosyl-} (1 $\rightarrow$ 4)-2- $O$-acetyl-3,6-di- $O$-benzoyl- $\alpha$-D-mannopyranosyl-(1 $\rightarrow 3)-[2,3,4$-tri- $O$-benzoyl- $\beta$-D-xylopyranosyl$(1 \rightarrow 2)]-4,6$-di- $O$-benzoyl- $\alpha$-D-mannopyranosyl- $(1 \rightarrow 3)$ [2,3,4-tri- $O$-benzoyl- $\beta$-D-xylopyranosyl-( $\rightarrow 2)]-4,6$-di$O$-benzoyl- $\alpha$-D-mannopyranoside (19)

To a cooled solution $\left(0^{\circ} \mathrm{C}\right)$ of $\mathbf{1 2}(1.83 \mathrm{~g}, 1.0 \mathrm{mmol})$ and $18(580 \mathrm{mg}, 0.7 \mathrm{mmol})$ in anhyd $\mathrm{CH}_{2} \mathrm{Cl}_{2}(10 \mathrm{~mL})$ was added TMSOTf $(8 \mu \mathrm{L}, 0.05 \mathrm{mmol})$. The mixture was stirred at this temperature for $2 \mathrm{~h}$, and then quenched with $\mathrm{Et}_{3} \mathrm{~N}$ (one drop). The solution was concentrated to give a residue. Purification of the residue by silica gel column chromatography (1:1.5 petroleum ether-EtOAc) gave $19(1.02 \mathrm{~g}, 60 \%)$ as a foamy solid: $[\alpha]_{\mathrm{D}}-26.9(c 0.5$, $\left.\mathrm{CHCl}_{3}\right) ;{ }^{1} \mathrm{H} \mathrm{NMR}\left(\mathrm{CDCl}_{3}, 400 \mathrm{MHz}\right): \delta 8.09-7.23(\mathrm{~m}$, $75 \mathrm{H}, 15 \mathrm{Ph} H), 5.82\left(\mathrm{dd}, 1 \mathrm{H}, J_{2,3} 3.2 \mathrm{~Hz}, J_{3,4} 10.0 \mathrm{~Hz}, \mathrm{H}-3\right.$ of $\operatorname{Man} p), 5.70\left(\mathrm{dd}, 1 \mathrm{H}, J_{2,3}=J_{3,4}=5.8 \mathrm{~Hz}, \mathrm{H}-3\right.$ of $\mathrm{Xyl} p), 5.66\left(\mathrm{dd}, 1 \mathrm{H}, J_{2,3}=J_{4,5}=7.0 \mathrm{~Hz}, \mathrm{H}-3\right.$ of Xylp), $5.54\left(\mathrm{dd}, 1 \mathrm{H}, J_{3,4}=J_{4,5}=10.0 \mathrm{~Hz}, \mathrm{H}-4\right.$ of $\left.\operatorname{Man} p\right), 5.50$ $\left(\mathrm{dd}, 1 \mathrm{H}, J_{2,3}=J_{3,4}=10.0 \mathrm{~Hz}, \mathrm{H}-4\right.$ of $\left.\operatorname{Man} p\right), 5.47(\mathrm{~m}$, $1 \mathrm{H}, \mathrm{H}-4$ of $\mathrm{Xyl} p), 5.47-5.36(\mathrm{~m}, 3 \mathrm{H}), 5.21-5.15(\mathrm{~m}, 2 \mathrm{H})$, $5.10(\mathrm{~s}, 1 \mathrm{H}, \mathrm{H}-1$ of $\operatorname{Man} p), 5.10\left(\mathrm{~d}, 1 \mathrm{H}, J_{1,2} 5.0 \mathrm{~Hz}, \mathrm{H}-1\right.$ of Xylp), $5.04\left(\mathrm{~d}, 1 \mathrm{H}, J_{1,2} 4.9 \mathrm{~Hz}, \mathrm{H}-1\right.$ of Xylp), $5.00(\mathrm{~m}$, 1H, H-4 of Xylp), 4.84 (s, 1H, H-1 of Manp), 4.80 (d, $1 \mathrm{H}, J_{1,2} 4.8 \mathrm{~Hz}, \mathrm{H}-1$ of Xylp), 4.55 (s, 1H, H-1 of Manp), 4.75-3.10 (m, 21H), $3.21\left(\mathrm{~s}, 3 \mathrm{H}, \mathrm{OCH}_{3}\right), 1.81(1 \mathrm{~s}, 3 \mathrm{H}$, $\left.\mathrm{COCH}_{3}\right) ; \quad{ }^{13} \mathrm{C} \quad \mathrm{NMR} \quad\left(100 \mathrm{MHz}, \quad \mathrm{CDCl}_{3}\right): \quad 168.9$ $\left(\mathrm{COCH}_{3}\right), 166.0,166.0,165.6,165.5,165.4,165.3,165.3$, $165.2,165.1,165.1,165.1,164.9,164.6,164.6,164.5$ (15C, 15COPh), 100.9, 100.1, 99.7, 99.6, 99.5, 98.8 (6C, C-1 $\left.{ }^{\mathrm{I}-\mathrm{IV}}\right), 78.88,77.57,77.26,76.85,75.17,74.46,70.55$, $70.21,70.18,70.10,69.93,69.77,69.62,69.30,69.23$, $69.11,68.97,68.40,68.32,64.36,64.00,62.13,61.40$, 60.95, $60.40(\mathrm{C}-2$ to $\mathrm{C}-6), 54.9\left(\mathrm{OCH}_{3}\right), 20.3\left(\mathrm{COCH}_{3}\right)$. Anal. Calcd for $\mathrm{C}_{141} \mathrm{H}_{120} \mathrm{O}_{44}$ : C, 67.25; H, 4.77. Found: C, 67.48; H, 4.66.

3.17. Methyl $\beta$-D-xylopyranosyl-( $1 \rightarrow 4)-\alpha$-D-mannopyranosyl-(1 $\rightarrow 3)$-[ $\beta$-D-xylopyranosyl-(1 $\rightarrow 2)]-\alpha-D-m a n n o-$ pyranosyl-( $1 \rightarrow 3)$-[ $\beta$-D-xylopyranosyl-(1 $\rightarrow 2)]-\alpha-D$-mannopyranoside (20)

Hexasaccharide 19 (100 mg, $0.04 \mathrm{mmol}$ ) was dissolved in a satd methanolic ammonia $(10 \mathrm{~mL})$. After stirring at $\mathrm{rt}$ for $72 \mathrm{~h}$, the reaction mixture was concentrated and purified on a Bio-Gel P2 column (eluent: water), affording the target hexasaccharide $20(30 \mathrm{mg}, 80 \%)$ as a foamy solid: $[\alpha]_{\mathrm{D}}+70.1\left(c \quad 0.5, \mathrm{H}_{2} \mathrm{O}\right) ;{ }^{1} \mathrm{H} \operatorname{NMR}\left(\mathrm{D}_{2} \mathrm{O}\right.$, $400 \mathrm{MHz}): \delta 5.09$ (s, 1H, H-1 of $\operatorname{Man} p), 5.00$ (s, 1H, H-1 of $\operatorname{Man} p), 4.75(\mathrm{~s}, 1 \mathrm{H}, \mathrm{H}-1$ of $\operatorname{Man} p), 4.32\left(\mathrm{~d}, 1 \mathrm{H}, J_{1,2}\right.$ $7.8 \mathrm{~Hz}, \mathrm{H}-1$ of Xylp), $4.30\left(\mathrm{~d}, 1 \mathrm{H}, J_{1,2} 7.9 \mathrm{~Hz}, \mathrm{H}-1\right.$ of $\mathrm{Xyl} p), 4.28\left(\mathrm{~d}, 1 \mathrm{H}, J_{1,2} 7.8 \mathrm{~Hz}, \mathrm{H}-1\right.$ of $\left.\mathrm{Xyl} p\right), 4.16-3.20$ $(\mathrm{m}, 36 \mathrm{H}) ;{ }^{13} \mathrm{C}$ NMR $\left(100 \mathrm{MHz}, \mathrm{D}_{2} \mathrm{O}\right): 103.6,103.5$, 103.4, 102.2, 100.5, 99.0 (6C, C- $\left.1^{\mathrm{I}-\mathrm{IV}}\right), 78.85,78.00$, $77.11,76.28,76.05,75.84,75.56,73.40,73.29,72.69$, $72.64,71.98,69.72,69.50,69.30,69.26,69.18,69.11$, 66.41, 66.09, 65.16, 64.99, 60.74, 60.23 (C-2 to C-6), 54.9 $\left(\mathrm{OCH}_{3}\right)$. Anal. Calcd for $\mathrm{C}_{34} \mathrm{H}_{58} \mathrm{O}_{28}$ : C, 44.64; H, 6.35. Found: C, 44.53; H, 6.42.

\subsection{Methyl 2,3,4-tri- $O$-benzoyl- $\beta$-D-xylopyranosyl-} (1 $\rightarrow$ 4)-3,6-di- $O$-benzoyl- $\alpha$-D-mannopyranosyl-( $1 \rightarrow 3)$ $[2,3,4$-tri- $O$-benzoyl- $\beta$-D-xylopyranosyl-(1 $\rightarrow 2)]-4,6$-di$O$-benzoyl- $\alpha$-D-mannopyranosyl-(1 $\rightarrow 3)-[2,3,4$-tri- $O$-benzoyl- $\beta$-D-xylopyranosyl-( $1 \rightarrow 2)]-4,6$-di- $O$-benzoyl- $\alpha$-Dmannopyranoside (21)

To a solution of $19(470 \mathrm{mg}, 0.17 \mathrm{mmol})$ in anhyd $\mathrm{CH}_{2} \mathrm{Cl}_{2}(10 \mathrm{~mL})$ was added anhyd $\mathrm{MeOH}(40 \mathrm{~mL})$, then $\mathrm{AcCl}(3.5 \mathrm{~mL})$ was added to the reaction mixture at $0{ }^{\circ} \mathrm{C}$. The mixture was stirred at $\mathrm{rt}$ for 3 days, TLC (1:1 petroleum ether-EtOAc) showed that the starting material disappeared. The solution was neutralized with $\mathrm{Et}_{3} \mathrm{~N}$, then concentrated to dryness. Purification of the residue by silica gel column chromatography (1:1.9 petroleum ether-EtOAc) gave $21(244 \mathrm{mg}, 60 \%)$ as a foamy solid: $[\alpha]_{\mathrm{D}}-37.5$ (c 1.0, $\left.\mathrm{CHCl}_{3}\right) ;{ }^{1} \mathrm{H}$ NMR 
(400 MHz, $\left.\mathrm{CDCl}_{3}\right): \delta 8.13-7.25(\mathrm{~m}, 75 \mathrm{H}, 15 \mathrm{PhH}), 5.70$ $\left(\mathrm{dd}, 1 \mathrm{H}, J_{3,4}=J_{4,5}=5.6 \mathrm{~Hz}, \mathrm{H}-3\right.$ of $\left.\mathrm{Xyl} p\right), 5.68(\mathrm{dd}$, $1 \mathrm{H}, J_{2,3} 3.3 \mathrm{~Hz}, J_{3,4} 10.1 \mathrm{~Hz}, \mathrm{H}-3$ of Manp), $5.62(\mathrm{dd}$, $1 \mathrm{H}, J_{2,3}=J_{3,4}=7.2 \mathrm{~Hz}, \mathrm{H}-3$ of $\left.\mathrm{Xyl} p\right), 5.52(\mathrm{dd}, 1 \mathrm{H}$, $J_{3,4}=J_{4.5}=10.2 \mathrm{~Hz}, \quad \mathrm{H}-4$ of Manp), $5.49(\mathrm{dd}, 1 \mathrm{H}$, $J_{3,4}=J_{4,5}=10.1 \mathrm{~Hz}, \mathrm{H}-4$ of $\left.\operatorname{Man} p\right), 5.40-5.36(\mathrm{~m}, 4 \mathrm{H})$, 5.19-5.15 (m, 2H), $5.10(\mathrm{~s}, 1 \mathrm{H}, \mathrm{H}-1$ of $\operatorname{Man} p), 5.03(\mathrm{~d}$, $1 \mathrm{H}, J_{1,2} 4.8 \mathrm{~Hz}, \mathrm{H}-1$ of Xylp), $5.00(\mathrm{~m}, 1 \mathrm{H}, \mathrm{H}-4$ of Xylp), $4.84\left(\mathrm{~d}, 1 \mathrm{H}, J_{1,2} 4.9 \mathrm{~Hz}, \mathrm{H}-1\right.$ of Xylp), $4.82(\mathrm{~s}, 1 \mathrm{H}, \mathrm{H}-1$ of $\operatorname{Man} p), 4.51\left(\mathrm{~d}, 1 \mathrm{H}, J_{1,2} 4.9 \mathrm{~Hz}, \mathrm{H}-1\right.$ of Xylp), 4.41 (s, $1 \mathrm{H}, \mathrm{H}-1$ of Manp), 4.75-3.10 (m, 22H), $3.20(\mathrm{~s}, 3 \mathrm{H}$, $\left.\mathrm{OCH}_{3}\right) ;{ }^{13} \mathrm{C} \mathrm{NMR}\left(100 \mathrm{MHz}, \mathrm{CDCl}_{3}\right): 165.9,165.9$, $165.5,165.5,165.4,165.3,165.2,165.1,165.0,165.0$, $165.0,164.8,164.8,164.4,164.4(15 \mathrm{C}, 15 \mathrm{COPh}), 102.0$, $100.9,100.9,99.7,99.7,98.7\left(6 \mathrm{C}, \mathrm{C}-1^{\mathrm{I}-\mathrm{IV}}\right), 78.73,77.59$, $77.20,76.27,74.87,77.17,72.20,70.70,70.47,70.29$, $70.10,69.88,69.81,69.50,69.35,69.13,69.08,68.97$, $68.90,68.83,68.21,64.21,63.80,62.14,61.40,60.97$, $60.83,60.22(\mathrm{C}-2$ to $\mathrm{C}-6), 54.7\left(\mathrm{OCH}_{3}\right)$. Anal. Calcd for $\mathrm{C}_{139} \mathrm{H}_{118} \mathrm{O}_{43}$ : C, 67.42; H, 4.77. Found: $\mathrm{C}, 67.20 ; \mathrm{H}$, 4.88 .

\subsection{Acetyl-transfer reaction and a trial with bromide 23 as the donor}

To a cooled solution $\left(0^{\circ} \mathrm{C}\right)$ of $21(120 \mathrm{mg}, 0.05 \mathrm{mmol})$ and $22(50 \mathrm{mg}, 0.10 \mathrm{mmol})$ in anhyd $\mathrm{CH}_{2} \mathrm{Cl}_{2}(5 \mathrm{~mL})$ was added TMSOTf $(2 \mu \mathrm{L}, 0.01 \mathrm{mmol})$. The mixture was stirred at this temperature for $2 \mathrm{~h}$, and then quenched with $\mathrm{Et}_{3} \mathrm{~N}$ (one drop). The solution was concentrated to give a residue. Purification of the residue by silica gel column chromatography (1:1.5 petroleum ether-EtOAc) gave a product $(40 \mathrm{mg})$ as a foamy solid, and its ${ }^{1} \mathrm{H}$ NMR data were identical with that of 19.

To a cooled solution $\left(0^{\circ} \mathrm{C}\right)$ of $21(120 \mathrm{mg}, 0.05 \mathrm{mmol})$ and $23(23 \mathrm{mg}, 0.06 \mathrm{mmol})$ in anhyd $\mathrm{CH}_{2} \mathrm{Cl}_{2}(5 \mathrm{~mL})$ and 2,4-lutidine $(6 \mu \mathrm{L}, 0.05 \mathrm{mmol})$, was added silver triflate
(14 $\mathrm{mg}, 0.06 \mathrm{mmol})$. The mixture was stirred at this temperature for $6 \mathrm{~h}$, and no reaction occurred.

\section{Acknowledgements}

This work was supported by The Chinese Academy of Sciences (KZCX3-J-08) and by The National Natural Science Foundation of China (Projects 30070185 and 39970864).

\section{References}

1. Cherniak, R. Top. Med. Mycol. 1988, 2, 40-54.

2. Bhattacharjee, A. K.; Bennett, J. E.; Glaudemans, C. P. J. Rev. Infect. Dis. 1982, 6, 619-624.

3. Wilson, D. E.; Bennett, J. E.; Bailey, J. W. Proc. Soc. Exp. Biol. Med. 1968, 127, 820-827.

4. (a) Bhattacharjee, A. K.; Kwon-Chung, K. J.; Glaudemans, C. P. J. Carbohydr. Res. 1979, 73, 183-192; (b) Bhattacharjee, A. K.; Kwon-Chung, K. J.; Glaudemans, C. P. J. Carbohydr. Res. 1981, 95, 237-245; (c) Bhattacharjee, A. K.; Kwon-Chung, K. J.; Glaudemans, C. P. J. Carbohydr. Res. 1980, 82, 103-111; (d) Bhattacharjee, A. K.; Kwon-Chung, K. J.; Glaudemans, C. P. J. Mol. Immunol. 1979, 16, 531-540.

5. (a) Garegg, P. J.; Olsson, L.; Oscarson, S. J. Carbohydr. Chem. 1993, 12, 195-203; (b) Garegg, P. J.; Olsson, L.; Oscarson, S. Bioorg. Med. Chem. 1996, 4, 1867-1878.

6. Zegelaar-Jaarsveld, K.; Smits, S. A. W.; van der Marel, G. A.; van Boom, J. H. Bioorg. Med. Chem. 1996, 4, 18191823 .

7. (a) Zhang, J.; Kong, F. Tetrahedron Lett. 2003, 18391850; (b) Zhang, J.; Kong, F. Bioorg. Med. Chem. 2003, 11, 4027-4037; (c) Zhang, J.; Kong, F. Carbohydr. Res. 2003, 338, 1719-1725.

8. Zhang, J.; Kong, F. Tetrahedron: Asymmetry 2002, 13, 243-252.

9. Chen, L.; Kong, F. Carbohydr. Res. 2002, 337, 2335-2341.

10. Schmidt, R. R.; Kinzy, W. Adv. Carbohydr. Chem. Biochem. 1994, 50, 21-125.

11. Byramova, N. E.; Ovchinnikov, M. V.; Backinowsky, L. V.; Kochetkov, N. K. Carbohydr. Res. 1983, 124, c8-c10. 